\title{
Ateliers interdisciplinaires sur les évaluations en milieu de travail
}

Les évaluations en milieu de travail (Mini-CEX et DOPS) font depuis peu partie intégrante des programmes de formation postgraduée des sociétés de discipline médicale. Sur mandat de l'Institut suisse pour la formation médicale postgraduée et continue (ISFM), l'Institut d'enseignement médical de l'Université de Berne (IML) proposera à nouveau des cours "Teach the teacher» en 2015. Ces ateliers interdisciplinaires permettent aux médecins-cadres de se familiariser avec les évaluations en milieu de travail et les techniques de feed-back.

\section{Les dates des prochains ateliers (en allemand)}

\section{Janvier 2015}

15.1 Zurich: 16h30-18h3o, USZ, Prof. H. Bucher 22.1 Bâle: 16h30-18h30, UHB, Prof. R. Bingisser

\section{Février 2015}

5.2 Zurich: 16h30-18h30, USZ, Prof. H. Bucher

\section{Mars 2015}

5.3 Lucerne: 16 hoo-18hoo, KSL, Dr Th. Hodel

\section{Avril 2015}

16.4 Berne: 16h30-18h3o, Hôpital de l'Ile, Prof. R. Greif 16.4 Bâle: 16h30-18h3o, UHB, Prof. R. Bingisser

Mai 2015

28.5 Berne: 16h30-18h3o, Hôpital de l'Ile, Prof. R. Greif

\section{Inscriptions}

Inscriptions via: http://aba.iml.unibe.ch/wb/teaching. php?lang=de ou auprès des coordinateurs de la formation postgraduée des hôpitaux concernés.
Die Arbeitsplatz-basierten Assessments Mini-CEX und DOPS sind seit kurzem integrierender Bestandteil der Weiterbildungsprogramme der Fachgesellschaften. Im Auftrag des Schweizerischen Instituts für ärztliche Weiter- und Fortbildung (SIWF) organisiert das Institut für Medizinische Lehre der Universität Bern (IML) deshalb auch 2015 wieder sogenannte "Teach the teacher»-Kurse. Diese interdisziplinären Workshops bieten Kaderärztinnen und -ärzten die Möglichkeit, sowohl die Assessments wie auch die Feedbacktechniken näher kennenzulernen.

\section{Daten der nächsten Workshops}

\section{Januar 2015}

15.1. Zürich: 16.30-18.30 Uhr, USZ, Prof. H. Bucher 22.1. Basel: $16.30-18.30$ Uhr, UHB, Prof. R. Bingisser

\section{Februar 2015}

5.2. Zürich: 16.30-18.30 Uhr, USZ, Prof. H. Bucher

\section{März 2015}

5.3. Luzern: 16.0o-18.0o Uhr, KSL, Dr. Th. Hodel

\section{April 2015}

16.4. Bern: 16.30-18.30 Uhr, Inselspital, Prof. R. Greif 16.4. Basel: 16.30-18.30 Uhr, UHB, Prof. R. Bingisser

\section{Mai 2015}

28.5. Bern: 16.30-18.30 Uhr, Inselspital, Prof. R. Greif

\section{Anmeldung}

Anmeldungen sind erbeten via: http://aba.iml.unibe. ch/wb/teaching.php?lang=de oder an die Weiterbildungskoordinatoren der entsprechenden Spitäler. 